Pacific Journal of Mathematics

THE POLARIZATION OF A LENS 


\title{
THE POLARIZATION OF A LENS
}

\author{
JOHN G. HERRIOT
}

1. Introduction. In a previous paper [3], the author obtained inequalities comparing the capacity of a lens with various geometric quantities of a lens. A lens may be described simply as a solid of revolution determined by the intersection of two spheres. Hore precisely, if $c>0$, the solid of revolution generated by revolving about the imaginary axis the area in the complex $z$-plane defined by the inequalities

$$
\theta_{1} \leq \arg \frac{z-c}{z+c} \leq \theta_{2}
$$

is called a lens. We may suppose $0<\theta_{1} \leq \theta_{2}<2 \pi$. It is, however, more convenient to characterize a lens in terms of its exterior angles. Accordingly we denote by $\alpha$ and $\beta$ the exterior angles which the two portions of the boundary of the generating area make with the real axis. It is easily seen that $\beta=\theta_{1}, \alpha=$ $2 \pi-\theta_{2}$. We shall assurse, as we may without loss of generality, that $\alpha \leq \beta$. The sum of these angles, $\alpha_{0}+\beta$, is called the dielectric angle of the lens. Clearly we have $\alpha+\beta \leq 2 \pi$, and hence we need consider only values of $\alpha$ not exceeding $\pi$. Sometimes it is convenient to introduce the radii $a$ and $b$ of the intersecting spheres; these are given by

$$
c=a \sin \alpha=b|\sin \beta|
$$

It is clear that when $\alpha+\beta=\pi$ the lens becomes a sphere; and when $\alpha+\beta \geq \pi$, $\beta \leq \pi$, the lens is convex. When $\beta \neq 0$ and $\alpha \rightarrow 0$, with $a$ fixed, the lens becomes a sphere of radius $a$. When $\alpha, \beta \rightarrow 0$ in such a manner that $\beta=k \%$, and $a$ is kept fixed, the lens becomes two tangent spheres of radii $a$ and $a / l$. When $\alpha, \beta \rightarrow \pi$, with $c$ fixed, the lens becomes a circular disk of radius $c$.

Received November 15, 1950. Presented to the American Mathematical Society, September 7, 1948 and October 28, 1950. The results presented in this paper were obtained in the course of research conducted under the sponsorship of the Office of Naval Research.

Pacific J. Math. 1 (1951), 369-397. 
In the present paper we consider the polarization of a lens, obtaining inequalities comparing the average polarization with the capacity and volume of the lens. This investigation is one phase of a general study of relationships between various physical and geometric quantities which has been carried on at Stanford University during the past four years under the direction of Professors Pólya and Szegö [5].

We now explain the concept of polarization as it has been defined by Schiffer and Szegö [6]. (Cf. also P'ólya and Szegö [5].) Consider an infinite electric field whose direction is determined by the unit vector $h$. When a conducting solid is placed in this field, the uniform field will be disturbed; the disturbance is equivalent to superimposing another field on the original one. If the electric potential of the superimposed field is denoted by $\psi$, then its energy is given, apart from trivial factors, by

$$
P=\iiint|\operatorname{grad} \psi|^{2} d \tau,
$$

the integral being extended over the whole space exterior to the solid.

We note that the function $\psi$ is harmonic and behaves like a dipole at infinity. Also $\psi$ satisfies on the surface of the given solid the boundary condition

$$
\psi=\mathrm{h} \cdot \mathrm{r}+\text { constant },
$$

where $\mathrm{r}$ is the radius vector. (The additive constant must be chosen properly.)

We call the quantity $P$ the polarization in the $h$-direction. It is easily verified that $P$ is a quadratic form in the components of $h$ :

$$
P=\sum_{i, k=1}^{3} P_{i, k} h_{i} h_{k}
$$

The coefficients of this form depend naturally on the coordinate system used; however, the invariants of this form are independent of the coordinate system. The simplest of these invariants, and the one with which we shall be concerned in this paper, is the average polarization $P_{m}$, defined by

$$
P_{1,1}+P_{2,2}+P_{3,3}=3 P_{m} \text {. }
$$

The study of $P$ is facilitated by introducing the expansion of the potential $\psi$ at infinity, where, as has already been observed, it behaves like a dipole. The strength component of this dipole in the direction $h$ can be represented in terms of the leading coefficient (that of $r^{-2}$ ) of the potential; it is a quadratic form 
in $h_{i}$, say

$$
E=\sum_{\imath, k=1}^{3} e_{\imath, k} h_{\imath} h_{k}
$$

We call it the dipole form associated with the polarization. By use of Green's formula, it is easy to establish the elegant relation

$$
P=4 \pi E-V,
$$

where $V$ is the volume of the solid. It has been found that $E$ shows a more regular behavior than $P$. We shall frequently find it convenient to consider $P_{m}+V$ in the present paper.

It is known that for a sphere we have $P=P_{m}=2 \mathrm{~V}$. It is conjectured that $P_{m}>2 \mathrm{~V}$ for other solids. Now for arbitrary solids it is well known that the capacity $C$ is not less than the radius of a sphere having the same volume as the given solid. (See e.g. Pólya and Szegö [4].) Thus we have $V \leq(4 \pi / 3) C^{3}$. Hience the inequality $P_{m} \geq(8 \pi / 3) C^{3}$ is stronger than $P_{m} \geq 2 \mathrm{~V}$. An even stronger inequality is

$$
P_{m}+V \geq 4 \pi C^{3}
$$

Since, as has already been pointed out, $E$ shows a more regular behavior than $P$, it is not surprising that this last inequality (2) is the easiest to investigate. It can be studied readily in the case of a lens by means of explicit expressions which Schiffer and Szegö [6] have given for $e_{x}, e_{y}, e_{z}\left(e_{1,1}, e_{2,2}, e_{3,3}\right)$, where the $z$-axis is in the direction of the axis of the lens. From these we can write at once the expressions for $P_{x}, P_{y}, P_{z}$, the polarizations in the $x-, y-$, and $z-$ directions. These formulas with others are collected together for convenience in $\$ 2$. We then prove in $\$ 3$ the strongest inequality (2) for the case of the spherical bowl (lens with $\alpha+\beta=2 \pi$ ). The same inequality is proven in $\S_{4}$ for the socalled Kelvin case (lens with $\alpha+\beta=\pi / 2$ ), in $\$ 5$ for the case of two tangent spheres, and in $\zeta 6$ for the symmetric lens. More detailed information concerning the behavior of the corresponding ratio is obtained for some of these cases.

2. Basic formulas. In this section we collect for convenience several formulas which will be useful in the later sections. Those for the polarization of the lens in the general case and in the several special cases are obtained from the paper of Schiffer and Szegö [6]. Those for the capacity are taken from a paper by Szego 
[7], which also gives references to the original literature; they are also collected together in the author's previous paper [3], and in the paper of Schiffer and Szegö [6].

For the polarization of the lens, we have

$$
P_{1,1}=P_{2,2}=P_{x}=P_{y}=4 \pi e_{x}-1, P_{3,3}=P_{z}=4 \pi e_{z}-V,
$$

where

$$
e_{x}=e_{y}=2 c^{3} \int_{-\infty}^{\infty}\left(q^{2}+\frac{1}{4}\right) \frac{\operatorname{sh} \pi q \operatorname{ch}(\alpha-\beta) q+\operatorname{sh}(\alpha+\beta-\pi) q}{\operatorname{sh}(\alpha+\beta) q \operatorname{ch} \pi q} d q,
$$

$$
\begin{aligned}
e_{z}=4 c^{3} \int_{-\infty}^{\infty} q^{2} & \frac{\operatorname{sh} \pi q \operatorname{ch}(\alpha-\beta) q+\operatorname{sh}(\pi-\alpha-\beta) q}{\operatorname{sh}(\alpha+\beta) q \operatorname{ch} \pi q} d q \\
& -\frac{4 c^{3}\left[\int_{-\infty}^{\infty} q \operatorname{th} \pi q \frac{\operatorname{sh}(\alpha-\beta) q}{\operatorname{sh}(\alpha+\beta) q} d q\right]^{2}}{\int_{-\infty}^{\infty} \frac{\operatorname{sh} \pi q \operatorname{ch}(\alpha-\beta) q+\operatorname{sh}(\alpha+\beta-\pi) q}{\operatorname{sh}(\alpha+\beta) q \operatorname{ch} \pi q} d q} .
\end{aligned}
$$

For the electrostatic capacity of the lens, we have

$$
C=c \int_{-\infty}^{\infty} \frac{\operatorname{sh} \pi q \operatorname{ch}(\alpha-\beta) q+\operatorname{sh}(\alpha+\beta-\pi) q}{\operatorname{sh}(\alpha+\beta) q \operatorname{ch} \pi q} d q .
$$

For the case of the spherical bowl, in which $\alpha+\beta=2 \pi$, these formulas yield

$$
\begin{gathered}
e_{x}=e_{y}=\frac{1}{2} c^{3}\left[f^{\prime \prime}(\alpha)+f^{\prime \prime}(\pi)+f(\alpha)\right], \\
e_{z}=c^{3}\left\{f^{\prime \prime}(\alpha)-f^{\prime \prime}(\pi)-\left[f^{\prime}(\alpha)\right]^{2} / f(\alpha)\right\},
\end{gathered}
$$

and

$$
C=c f(\alpha)
$$

where

$$
f(\alpha)=\int_{-\infty}^{\infty}\left[\frac{\operatorname{ch}(\pi-\alpha) q}{\operatorname{ch} \pi q}\right]^{2} d q=\frac{1}{\pi}\left(1+\frac{\pi-\alpha}{\sin \alpha}\right) .
$$


We note that $f^{\prime \prime}(\pi)=(3 \pi)^{-1}$.

For the case of the lens with $\hat{u}+\beta=\pi / 2$ (Kelvin case), we have

$$
\begin{aligned}
& e_{x}=e_{y}=\frac{1}{2} c^{3}\left[k(\alpha)+k(\beta)-k(0)+k^{\prime \prime}(\alpha)+k^{\prime \prime}(\beta)-k^{\prime \prime}(0)\right], \\
& e_{z}=c^{3}\left\{k^{\prime \prime}(\alpha)+k^{\prime \prime}(\beta)+k^{\prime \prime}(0)-\left[k^{\prime}(\alpha)-k^{\prime}(\beta)\right]^{2} /[k(\alpha)+k(\beta)-k(0)]\right\},
\end{aligned}
$$

and

$$
C=c[k(\alpha)+k(\beta)-k(0)]=a+b-c,
$$

where

$$
k(\alpha)=\int_{-\infty}^{\infty} \frac{\operatorname{ch} 2 \alpha q}{\operatorname{ch} \pi q} d q=\sec \alpha
$$

[See formula (A-1) in Appendix A.].

For the limiting case of two tangent spheres of radii $a$ and $b$, we have

$$
\begin{array}{r}
e_{x}=e_{y}=\frac{1}{2}\left(\frac{a b}{a+b}\right)^{3}\left[2 \psi^{\prime \prime}(1)-\psi^{\prime \prime}\left(\frac{a}{a+b}\right)-\psi^{\prime \prime}\left(\frac{b}{a+b}\right)\right], \\
e_{z}=\left(\frac{a b}{a+b}\right)^{3}\left\{-2 \psi^{\prime \prime}(1)-\psi^{\prime \prime}\left(\frac{a}{a+b}\right)-\psi^{\prime \prime}\left(\frac{b}{a+b}\right)\right. \\
\left.-\frac{\left[\psi^{\prime}\left(\frac{a}{a+b}\right)-\psi^{\prime}\left(\frac{b}{a+b}\right)\right]^{2}}{-\psi\left(\frac{a}{a+b}\right)-\psi\left(\frac{b}{a+b}\right)-2 \gamma}\right\},
\end{array}
$$

and

$$
C=\frac{a b}{a+b}\left[-\psi\left(\frac{a}{a+b}\right)-\psi\left(\frac{b}{a+b}\right)-2 \gamma\right],
$$

where $\psi(u)=\Gamma^{\prime}(u) / \Gamma(u), \Gamma(u)$ being Euler's gamma function, and where $\gamma$ is Euler's constant.

Finally, for the case of the symmetric lens we obtain 


$$
\begin{gathered}
e_{x}=e_{y}=2 c^{3} \int_{-\infty}^{\infty}\left(q^{2}+\frac{1}{4}\right)(1-\operatorname{th} \pi q \operatorname{th} \alpha q) d q, \\
e_{z}=4 c^{3} \int_{-\infty}^{\infty} q^{2}(\text { th } \pi q \operatorname{cth} \alpha q-1) d q,
\end{gathered}
$$

and

$$
C=c \int_{-\infty}^{\infty}(1-\operatorname{th} \pi q \text { th } a q) d q
$$

An elementary calculation shows that the volume of the lens is given by

$$
V=\frac{\pi c^{3}}{6}\left[(2-\cos \alpha) \cot \frac{\alpha}{2} \csc ^{2} \frac{\alpha}{2}+(2-\cos \beta) \cot \frac{\beta}{2} \csc ^{2} \frac{\beta}{2}\right] \text {. }
$$

3. Spherical bowl, $\alpha+\beta=2 \pi$. The volume $V$ of the spherical bowl is clearly zero, so that the inequality (2) becomes $P_{m} \geq 4 \pi C^{3}$. In this section we consider the ratio $P_{m} / 4 \pi C^{3}$.

From (1), (3), (7), (8), and (9) we obtain

$$
\frac{P_{m}}{4 \pi C^{3}}=\frac{2 f^{\prime \prime}(\alpha) f(\alpha)+[f(\alpha)]^{2}-\left[f^{\prime}(\alpha)\right]^{2}}{3[f(\alpha)]^{4}} .
$$

If we make use of equation (10), which gives $f(\alpha)$ explicitly, and substitute $\delta$ for $\pi-\alpha$, we easily obtain

$$
\begin{aligned}
\frac{P_{m}}{4 \pi C^{3}}=\frac{\pi^{2}}{3(\delta+\sin \delta)^{4}}\left[3 \delta^{2}+4 \delta\right. & \sin \delta-\delta \sin 2 \delta \\
& \left.-\frac{1}{4} \sin ^{2} 2 \delta-2 \sin \delta \sin 2 \delta\right] .
\end{aligned}
$$

We differentiate (23) with respect to $\delta$ and find

$$
\frac{d}{d \delta}\left(\frac{P_{m}}{4 \pi C^{3}}\right)=-\frac{\pi^{2}(1+\cos \delta)^{2}}{3(\delta+\sin \delta)^{5}} H(2 \delta),
$$

where

$$
H(\delta)=\delta^{2}+\delta \sin \delta-4(1-\cos \delta) .
$$

We now proceed to show that $H(\delta) \geq 0$ for $0 \leq \delta \leq 2 \pi$. Clearly $H(0)=0$. Also, 


$$
\begin{aligned}
& H^{\prime}(\delta)^{\prime}=2 \delta+\delta \cos \delta-3 \sin \delta, \\
& H^{\prime \prime}(\delta)=2 \sin \delta\left(\tan \frac{1}{2} \delta-\frac{1}{2} \delta\right) .
\end{aligned}
$$

If $0 \leq \delta \leq \pi$, then $\sin \delta \geq 0$ and $\tan (\delta / 2) \geq \delta / 2$, so that $I^{\prime \prime}(\delta) \geq 0$. But if $\pi \leq \delta \leq 2 \pi$, then $\sin \delta \leq 0$ and $\tan (\delta / 2) \leq 0$, so that again $H^{\prime \prime}(\delta) \geq 0$. Thus $H^{\prime}(\delta)$ increases monotonely as $\delta$ increases from 0 to $2 \pi$. But $I^{\prime}(0)=0$. Thus $H^{\prime}(\delta) \geq 0$. Since $H(0)=0$, it follows that $H(\delta) \geq 0$ for $0 \leq \delta \leq 2 \pi$. From (24) it follows that $P_{m} / 4 \pi C^{3}$ decreases monotonely as $\delta$ increases from 0 to $\pi$. But from (23) we easily find that $P_{m} / 4 \pi C^{3}$ has the value $\pi^{2} / 9$ for $\delta=0$ and the value 1 for $\delta=\pi$. It follows that $P_{m} / 4 \pi C^{3}$ increases monotonely from 1 to $\pi^{2} / 9 \cong 1.097$ as $\alpha$ increases from 0 (sphere) to $\pi$ (circular disk). Thus for the spherical bowl the inequality $P_{m} \geq 4 \pi C^{3}$ is proven.

4. Kelvin case, $\alpha+\beta=\pi / 2$. We now consider the case of a lens of dielectric angle $\pi / 2$ formed by the intersection of orthogonal spheres. The polarization and capacity can again be expressed in terms of elementary functions, so that the study of the ratio $\left(P_{m}+V\right) / 4 \pi C^{3}$ is not difficult. For this case we use equations (1), (3), (11), (12), and (13) to obtain

$$
\begin{aligned}
& \frac{P_{m}+V}{4 \pi C^{3}}=\frac{2\left[k^{\prime \prime}(\alpha)+k^{\prime \prime}(\beta)\right][k(\alpha)+k(\beta)-k(0)]}{3[k(\alpha)+k(\beta)-k(0)]^{4}} \\
& +\frac{[k(\alpha)+k(\beta)-k(0)]^{2}-\left[k^{\prime}(\alpha)-k^{\prime}(\beta)\right]^{2}}{3[k(\alpha)+k(\beta)-k(0)]^{4}}
\end{aligned}
$$

where $k(\alpha)$ is given by (14), and, as throughout this section, $\beta=\pi / 2-\alpha$. We note that $k(\alpha)+k(\beta)-k(0)$ becomes infinite when $\alpha$ tends to zero or $\pi / 2$; in order to obtain a fraction whose numerator and denominator remain finite, it is convenient to multiply the numerator and denominator in (26) by $\sin ^{4} \alpha \cos ^{4} \alpha$. If we subtract 1 from both sides of (26), we obtain

$$
\frac{\rho_{m}+V}{4 \pi C^{3}}-1=\frac{k^{*}(\alpha)}{3 \sin ^{4} \alpha \cos ^{4} \alpha[k(\alpha)+k(\beta)-k(0)]^{4}},
$$

where 
(28)

$$
\begin{aligned}
& k^{*}(\alpha)= \sin ^{4} \alpha \cos ^{4} \alpha\left\{2\left[k^{\prime \prime}(\alpha)+k^{\prime \prime}(\beta)\right][k(\alpha)+k(\beta)-k(0)]\right. \\
&+[k(\alpha)+k(\beta)-k(0)]^{2}-\left[k^{\prime}(\alpha)-k^{\prime}(\beta)\right]^{2} \\
&\left.-3[k(\alpha)+k(\beta)-k(0)]^{4}\right\} .
\end{aligned}
$$

We note that $k^{*}(\alpha)$ is always finite. In order to prove the inequality (2), it suffices to prove that $k^{*}(\alpha) \geq 0$ for $0 \leq \alpha \leq \pi / 4$ since, as was pointed out earlier, we can always suppose $\alpha \leq \beta$. We make use of (14) to obtain the following necessary expressions:

$$
\begin{gathered}
\sin \alpha \cos \alpha[k(\alpha)+k(\beta)-k(0)]=\sin \alpha+\cos \alpha(1-\sin \alpha), \\
\sin ^{2} \alpha \cos ^{2} \alpha\left[k^{\prime}(\alpha)-k^{\prime}(\beta)\right]=\sin ^{3} \alpha-\cos ^{3} \alpha
\end{gathered}
$$

If we substitute (29), (30), and (31) in (28) we obtain, after some simplification, $k_{i}^{*}(\alpha)=2 \sin \alpha \cos \alpha(1-\cos \alpha)^{2}(1-\sin \alpha)^{2}\left[\cos \alpha(4-\sin \alpha)+2\left(1+2 \sin \alpha_{2}\right)\right]$. It is clear that each factor in this product is nonnegative, and hence $k^{*}(\alpha) \geq 0$ for $0 \leq \alpha \leq \pi / 4$ and indeed for $0 \leq \alpha \leq \pi / 2$. As previously noted, this is sufficient to prove the inequality (2) for this case.

5. Two tangent spheres. We now consider two tangent spheres of radii $a$ and b. (We assume without loss of generality that $b \leq a$.) We write $b /(a+b)=z$ ( $z$ should not be confused with the $z$-coordinate), and make use of (1), (3), (15), (16), and (17), obtaining

$$
\begin{array}{r}
\frac{P_{m}+V}{4 \pi c^{3}}=\frac{2\left[-\psi^{\prime \prime}(z)-\psi^{\prime \prime}(1-z)\right][-\psi(z)-\psi(1-z)-2 \gamma]}{3[-\psi(z)-\psi(1-z)-2 \gamma]^{4}} \\
-\frac{\left[\psi^{\prime}(z)-\psi^{\prime}(1-z)\right]^{2}}{3[-\psi(z)-\psi(1-z)-2 \gamma]^{4}} .
\end{array}
$$

Recalling that

$$
\psi(z)=-\gamma-\frac{1}{z}+\sum_{n=1}^{\infty}\left(\frac{1}{n}-\frac{1}{n+z}\right)
$$

we obtain 
(33)

$$
-\psi(z)-\psi(1-z)-2 \gamma=\frac{1}{z(1-z)}+\sum_{n=1}^{\infty}\left[\frac{2 n+1}{n^{2}+n+z(1-z)}-\frac{2}{n}\right]
$$

We note that this expression is a function of $z(1-z)$, and make the substitution $z(1-z)=y$. It is clear that $z$ lies between 0 and $1 / 2$, and hence $y$ lies between 0 and $1 / 4$. For such values of $y, z$ is a single-valued function of $y$ given by $2 z$ $=1-(1-4 y)^{1 / 2}$. We have also

$$
\frac{d y}{d z}=1-2 z=(1-4 y)^{1 / 2} \text {. }
$$

It follows that both $\psi^{\prime}(z)-\psi^{\prime}(1-z)$ and $-\psi^{\prime \prime}(z)-\psi^{\prime \prime}(1-z)$ are single-valued functions of $y$ for $0 \leq y \leq 1 / 4$. Thus, by (32), the same is true of $\left(P_{m}+V\right) / 4 \pi C^{3}$. We denote this function by $t(y)$. We shall show that $t(y)$ increases as $y$ increases. We therefore consider $t^{\prime}(y)$. A simple calculation gives

$$
t^{\prime}(y)=\frac{M}{3(1-4 y)^{1 / 2}[-\psi(z)-\psi(1-z)-2 \gamma]^{5}} .
$$

where

$$
\begin{aligned}
M=4\left\{\psi^{\prime}(z)-\psi^{\prime}(1-z)\right\} & \left\{2\left[-\psi^{\prime \prime}(z)-\psi^{\prime \prime}(1-z)\right][-\psi(z)-\psi(1-z)-2 \gamma]\right. \\
- & {\left.\left[\psi^{\prime}(z)-\psi^{\prime}(1-z)\right]^{2}\right\} } \\
& -2\left\{\psi^{\prime \prime \prime}(z)-\psi^{\prime \prime \prime}(1-z\}\{-\psi(z)-\psi(1-z)-2 \gamma\}^{2} .\right.
\end{aligned}
$$

If we make the substitution $z(1-z)=y$ in (33), and let $1 /[n(n+1)]=a_{n}$, we obtain

$$
y[-\psi(z)-\psi(1-z)-2 y]=1+y \sum_{n=1}^{\infty}\left[\frac{(2 n+1) a_{n}}{1+a_{n} y}-\frac{2}{n}\right] .
$$

But

$$
\begin{aligned}
\frac{(2 n+1) a_{n}}{1+a_{n} y}-\frac{2}{n}=-a_{n} & -(2 n+1) a_{n}^{2} y+(2 n+1) a_{n}^{3} y^{2} \\
& -\cdots+(-1)^{m-1}(2 n+1) a_{n}^{m} y^{m-1}+\cdots .
\end{aligned}
$$


It is easily verified that

$$
\sum_{n=1}^{\infty} a_{n}=\sum_{n=1}^{\infty}(2 n+1) a_{n}^{2}=1
$$

For convenience we let

$$
b_{m}=\sum_{n=1}^{\infty}(2 n+1) a_{n}^{m} \quad(m=2,3,4, \cdots) .
$$

By (36), we have $b_{2}=1$. It follows that

$$
y[-\psi(z)-\psi(1-z)-2 \gamma]=1-y-y^{2}+\sum_{m=3}^{\infty}(-1)^{m-1} b_{m} y^{m} .
$$

Now

$$
b_{m}=\sum_{n=1}^{\infty}(2 n+1) a_{n}^{m-1} \frac{1}{n(n+1)} \leq \frac{1}{2} b_{m-1} \quad(m=3,4,5, \cdots) \text {. }
$$

Repeated application of this inequality shows that

$$
b_{m} \leq \frac{1}{2^{m-2}} b_{2}=\frac{1}{2^{m-2}} .
$$

Thus the series in equation (38) certainly converges uniformly and absolutely for $0 \leq y \leq 1 / 4$.

If we divide equation (38) by $y$, differentiate with respect to $z$, and make use of (34), we obtain

$$
\begin{aligned}
y^{2}\left[\psi^{\prime}(z)\right. & \left.-\psi^{\prime}(1-z)\right] \\
& =(1-4 y)^{3 / 2}\left[1+y^{2}+\sum_{m=3}^{\infty}(-1)^{m}(m-1) b_{m} y^{m}\right] .
\end{aligned}
$$

Similarly we find that 
(42) $y^{3}\left[-\psi^{\prime \prime}(z)-\psi^{\prime \prime}(1-z)\right]$

$$
=2\left\{1-3 y+\sum_{m=3}^{\infty}(-1)^{m-1}(m-2) \cdot\left[\frac{m-1}{2} b_{m}+(2 m-5) b_{m-1}\right] y^{m}\right\},
$$

and

$$
y^{4}\left[\psi^{\prime \prime \prime}(z)-\psi^{\prime \prime \prime}(1-z)\right]=6(1-4 y)^{1 / 2} Q,
$$

where

$$
Q=1-2 y+\sum_{m=4}^{\infty}(-1)^{m} \frac{(m-2)(m-3)}{3} \cdot\left[\frac{m-1}{2} b_{m}+(2 m-5) b_{m-1}\right] y^{m} \text {. }
$$

By means of (40) it is easily seen that the series in (41), (42), and (43) are uniformly and absolutely convergent for $0 \leq y \leq 1 / 4$. Moreover, the terms of these series as well as those of the series in (38) alternate in sign after the first few terms. If we make use of (39) and (40), we easily verify that the terms in each of these series decrease in absolute value for $0 \leq y \leq 1 / 4$. Consequently, each of these series may be conveniently estimated by taking a finite number of its terms. In order to simplify the estimates we need a better estimate for $b_{m}$ than is given by (40). We easily find that

$$
\frac{1}{2^{m-1}}<\frac{3}{2^{m}}<b_{m}<\frac{3}{2^{m}}+\frac{1}{2^{2 m-3}}<\frac{1}{2^{m-2}} \quad(m=3,4,5, \cdots) .
$$

The following estimates are then obtained:

$$
\begin{gathered}
y[-\psi(z)-\psi(1-z)-2 \gamma] \leq 1-y-y^{2}+b_{3} y^{3}-b_{4} y^{4}+\frac{1}{8} y^{5} \\
y[-\psi(z)-\psi(1-z)-2 \gamma] \\
\geq 1-y-y^{2}+b_{3} y^{3}-b_{4} y^{4}+\frac{1}{16} y^{5}-\frac{1}{16} y^{6}
\end{gathered}
$$

$$
\begin{aligned}
& y^{2}\left[\psi^{\prime}(z)-\psi^{\prime}(1-z)\right] \\
& \quad \leq(1-4 y)^{1 / 2}\left[1+y^{2}-2 b_{3} y^{3}+3 b_{4} y^{4}-\frac{1}{4} y^{5}+\frac{1}{4} y^{5}\right],
\end{aligned}
$$

$$
\begin{aligned}
y^{2}\left[\psi^{\prime}(z)\right. & \left.-\psi^{\prime}(1-z)\right] \\
& \geq(1-4 y)^{1 / 2}\left[1+y^{2}-2 b_{3} y^{3}+3 b_{4} y^{4}-\frac{1}{2} y^{5}\right],
\end{aligned}
$$


(49)

$$
\begin{aligned}
y^{3}\left[-\psi^{\prime \prime}(z)\right. & \left.-\psi^{\prime \prime}(1-z)\right] \\
& \geq 2\left[1-3 y+\left(b_{3}+1\right) y^{3}-3\left(b_{4}+2 b_{3}\right) y^{4}+3 y^{5}-4 y^{6}\right],
\end{aligned}
$$

$$
\begin{aligned}
y^{4}\left[\psi^{\prime \prime \prime}(z)\right. & \left.-\psi^{\prime \prime \prime}(1-z)\right] \\
& \leq 6(1-4 y)^{1 / 2}\left[1-2 y+\left(b_{4}+2 b_{3}\right) y^{4}-2 y^{5}+4 y^{5}\right] .
\end{aligned}
$$

All of these estimates are valid for $0 \leq y \leq 1 / 4$.

Before substituting these estimates in (35), we find it convenient to estimate certain combinations which appear there. From (49), (46), and (47) we obtain

$$
\begin{aligned}
y^{4} & \left\{2\left[-\psi^{\prime \prime}(z)-\psi^{\prime \prime}(1-z)\right][-\psi(z)-\psi(1-z)-2 \gamma]-\left[\psi^{\prime}(z)-\psi^{\prime}(1-z)\right]^{2}\right\} \\
& \geq 3-12 y+6 y^{2}+12\left(b_{3}+2\right) y^{3}-\left(22 b_{4}+56 b_{3}+5\right) y^{4} \\
& +\left(48 b_{4}+24 b_{3}+\frac{51}{4}\right) y^{5}+\left(6 b_{4}+12 b_{3}-\frac{63}{2}\right) y^{6} \\
& +\left(20 b_{4}-4 b_{3} b_{4}-8 b_{3}^{2}+\frac{29}{4}\right) y^{7}+\left(\frac{45}{4} b_{3}-24 b_{3} b_{4}+3 b_{4}^{2}+\frac{55}{4}\right) y^{8} \\
& -\left(\frac{45}{4} b_{4}+\frac{51}{4} b_{3}-36 b_{4}^{2}-\frac{7}{4}\right) y^{9}+\left(\frac{37}{4} b_{4}-\frac{5}{2} b_{3}+\frac{11}{16}\right) y^{10} \\
& -\left(\frac{11}{8}-6 b_{4}\right) y^{11}+\frac{7}{16} y^{12}+\frac{1}{4} y^{13} \\
& \geq 3-12 y+6 y^{2}+12\left(b_{3}+2\right) y^{3}-\left(22 b_{4}+56 b_{3}+5\right) y^{4}+24 y^{5}-28 y^{6}
\end{aligned}
$$

In passing to the lasst inequality we have made use of the inequalities (44) to estimate the coefficients of $y^{5}$ and $y^{6}$ and to prove that the sum of the last seven terms is nonnegative for $0 \leq y \leq 1 / 4$. From (45) we find in a similar way that

$$
\begin{aligned}
y^{2} & {[-\psi(z)-\psi(1-z)-2 \gamma]^{2} } \\
& \leq 1-2 y-y^{2}+2\left(b_{3}+1\right) y^{3}-\left(2 b_{4}+2 b_{3}-1\right) y^{4}+\frac{1}{2} y^{6} .
\end{aligned}
$$

We proceed in a similar manner using (48) and (51) to obtain

(53) $4 y^{6}\left\{\psi^{\prime}(z)-\psi^{\prime}(1-z)\right\}\left\{2\left[-\psi^{\prime \prime}(z)-\psi^{\prime \prime}(1-z)\right][-\psi(z)-\psi(1-z)-2 \gamma]\right.$

$$
\begin{aligned}
& \left.-\left[\psi^{\prime}(z)-\psi^{\prime}(1-z)\right]^{2}\right\} \\
& \geq(1-4 y)^{1 / 2}\left[12-48 y+36 y^{2}+24\left(b_{3}+2\right) y^{3}\right. \\
& \left.-\left(52 b_{4}+128 b_{3}-4\right) y^{4}+150 y^{5}-344 y^{6}\right] .
\end{aligned}
$$


The last combination which we shall need is obtained from (50) and (52); it is

$$
\begin{array}{r}
2 y^{6}\left[\psi^{\prime \prime \prime}(z)-\psi^{\prime \prime \prime}(1-z)\right][-\psi(z)-\psi(1-z)-2 \gamma]^{2} \\
\leq(1-4 y)^{1 / 2}\left[12-48 y+36 y^{2}+24\left(b_{3}+2\right) y^{3}\right. \\
\left.-12\left(b_{4}+4 b_{3}+3\right) y^{4}-42 y^{5}+95 y^{5}\right] .
\end{array}
$$

If we substitute from (53) and (54) into (35), and use the result that

$$
b_{4}+2 b_{3}=\sum_{n=1}^{\infty}(2 n+1)\left(a_{n}^{4}+2 a_{n}^{3}\right)=\sum_{n=1}^{\infty}\left[\frac{1}{n^{4}}-\frac{1}{(n+1)^{4}}\right]=1 \text {, }
$$

we obtain

$$
t^{\prime}(y) \geq \frac{192-439 y}{3 y[-\psi(z)-\psi(1-z)-2 \gamma]^{5}} .
$$

Now for $0 \leq y \leq 1 / 4$ it is clear from (38) that $y[-\psi(z)-\psi(1-z)-2 \gamma]$ is finite and positive. Hence by (55) we see that $t^{\prime}(y)>0$ for $0<y \leq 1 / 4$. It is easily verified from (35) that $t^{\prime}(0)=0$. Thus $t(y)$ increases monotonely as $y$ increases from 0 to $1 / 4$. This means that the ratio $\left(P_{m}+V\right) / 4 \pi C^{3}$ increases monotonely as $b$ increases from $U$ to $a$, where $a$ and $b$ are the radii of the tangent spheres.

Now we see that $b \rightarrow 0$ implies $z \rightarrow 0$ and hence $y \rightarrow 0$. If we multiply the numerator and denominator of (32) by $y^{4}$ and make use of (38), (41), and (42), we find that $b \rightarrow 0$ implies

$$
\frac{P_{m}+V}{4 \pi C^{3}} \rightarrow \frac{2(2)(1)-1}{3}=1
$$

Also we see that $z=1 / 2$ when $a=b$, and hence in this case (32) yields

$$
\frac{P_{m}+V}{4 \pi C^{3}}=\frac{-4 \psi^{\prime \prime}\left(\frac{1}{2}\right)}{3\left[-2 \psi\left(\frac{1}{2}\right)-2 \gamma\right]^{3}}=\frac{-\psi^{\prime \prime}\left(\frac{1}{2}\right)}{48 \log ^{3} 2}=\frac{7 \zeta(3)}{24 \log ^{3} 2} \cong 1.053,
$$

where $\zeta(z)$ denotes the Riemann zeta-function. (To obtain the values of $\psi^{\prime \prime}(1 / 2)$ and $\psi(1 / 2)$ see, for example, Copson [2, p. 229].)

Thus as $b$ increases from 0 (one sphere) to $a$ (equal spheres) we see that the ratio $\left(P_{m}+V\right) / 4 \pi C^{3}$ increases monotonely from 1 to $7 \zeta(3) /\left(24 \log ^{3} 2\right) \cong 1.053$. Thus we have proved the inequality $(2)$ for the case of tangent spheres. 
Of course the weaker inequalities $P_{m} \geq(8 \pi / 3) C^{3}$ and $P_{m} \geq 2 \mathrm{~V}$ follow immediately from (2). However, it is instructive to consider the behavior of the corresponding ratios for this case of tangent spheres. This behavior can be deduced from the results just obtained if we first study the behavior of $4 \pi C^{3} / 3 \mathrm{~V}$. It has already been pointed out that this ratio is never less than unity [4]

The volume may be obtained from (21) by setting $\beta=(a / b) \alpha$ and letting $\alpha \rightarrow 0$, or more simply by direct calculation. It is found to be

$$
\begin{aligned}
V & =\frac{4 \pi}{3}\left(a^{3}+b^{3}\right)=\frac{4 \pi}{3}(a+b)\left(a^{2}-a b+b^{2}\right) \\
& =\frac{4 \pi}{3}(a+b)^{3}\left(1-\frac{3 a b}{(a+b)^{2}}\right)=\frac{4 \pi}{3}(a+b)^{3}(1-3 y),
\end{aligned}
$$

since $y=z(1-z), z=b /(a+b)$.

If we now make use of (17) and (56) we find that

$$
\left(\frac{4 \pi c^{3}}{3 V}\right)^{1 / 3}=\frac{y[-\psi(z)-\psi(1-z)-2 \gamma]}{(1-3 y)^{1 / 3}} .
$$

This is a function of $y$ and we could differentiate it with respect to $y$ and prove the derivative nonnegative by a method similar to that used in treating $t^{\prime}(y)$ above. But the following method seems to be more elegant. We have

$$
(1-3 y)^{-1 / 3}=1+\sum_{m=1}^{\infty} \frac{1 \cdot 4 \cdot 7 \cdot \cdots \cdot(3 m-2)}{m !} y^{m} \text {. }
$$

If we substitute (58) and (38) into (57), we obtain

$$
\left(\frac{4 \pi C^{3}}{3 V}\right)^{1 / 3}=1+\sum_{m=3}^{\infty} h_{m} y^{m}
$$

where

$$
h_{m}=\frac{1 \cdot 4 \cdot 7 \cdot \cdots \cdot(3 m-2)}{m !}-\frac{1 \cdot 4 \cdot 7 \cdot \cdots \cdot(3 m-5)}{(m-1) !}-\frac{1 \cdot 4 \cdot 7 \cdot \cdots \cdot(3 m-8)}{(m-2) !}
$$




$$
\begin{aligned}
& +\sum_{\mu=3}^{m-1}(-1)^{\mu-1} \frac{1 \cdot 4 \cdot 7 \cdot \cdots \cdot(3 m-3 \mu-2)}{(m-\mu) !} b_{\mu}+(-1)^{m-1} b_{m} \\
= & \frac{1 \cdot 4 \cdot 7 \cdot \cdots \cdot(3 m-8)(5)(m-2)(m-1)}{m !} \\
& +\sum_{\mu=3}^{m-1}(-1)^{\mu-1} \frac{1 \cdot 4 \cdot 7 \cdot \cdots \cdot(3 m-3 \mu-2)}{(m-\mu) !} b_{\mu}+(-1)^{m-1} b_{m} .
\end{aligned}
$$

The first term in this last expression for $h_{m}$ is positive, and the rest of the terms are alternately positive and negative and decrease in absolute value. It follows that $h_{m}>0$. Thus $\left(4 \pi C^{3} / 3 V\right)^{1 / 3}$ increases as $y$ increases. The same is therefore true of $4 \pi C^{3} / 3 \mathrm{~V}$. Now (57) shows that when $b \rightarrow 0$, that is, when $y \rightarrow 0$, this ratio tends to one. When $b=a$, we have $y=1 / 4$, and (57) shows that the ratio $4 \pi C^{3} / 3 V$ is $4 \log ^{3} 2 \cong 1.332$. Thus as $b$ increases from 0 to $a$ we see that the ratio $4 \pi C^{3} / 3 V$ increases monotonely from 1 to $4 \log ^{3} 2$.

Combining with our previous result we conclude that the ratio $\left(P_{m}+V\right) / 3 V$ increases monotonely from 1 to $(7 / 6) \zeta(3) \cong 1.402$ as $b$ increases from 0 to $a$. Now since

$$
\frac{P_{m}}{2 V}=\frac{3}{2} \frac{P_{m}+V}{3 V}-\frac{1}{2}
$$

it is clear that $P_{m} / 2 V$ increases monotonely from 1 to $(7 / 4) \zeta_{3}(3)-1 / 2 \cong 1.604$ as $b$ increases from 0 to $a$. Finally since

$$
\frac{P_{m}}{(8 \pi / 3) C^{3}}=\frac{3}{2} \frac{P_{m}+V}{4 \pi C^{3}}-\frac{1}{2} \frac{3 V}{4 \pi C^{3}},
$$

we see that $P_{m} /\left[(8 \pi / 3) C^{3}\right]$ increases monotonely from one to the quantity $[7 \zeta(3)-2] /\left(16 \log ^{3} 2\right) \cong 1.204$ as $b$ increases from zero to $a$.

6. Symmetric lens. In this section we prove the inequality (2) for the case of the symmetric lens. If we make use of (1), (3), (18), (19), and (20) we find that

$$
\frac{P_{m}+V}{4 \pi \mathrm{L}^{3}}=\frac{\int_{-\infty}^{\infty}(1-\operatorname{th} \pi q \operatorname{th} \alpha q) d q+8 \int_{-\infty}^{\infty} \frac{q^{2} \operatorname{th} \pi q}{\operatorname{sh} 2 \alpha q} d q}{3\left[\int_{-\infty}^{\infty}\left(1-\operatorname{th} \pi q \text { th } a_{q}\right) d q\right]^{3}} .
$$


We denote this ratio by $S(\alpha)$. We note that $S(0)=7 \zeta(3) /\left(24 \log ^{3} 2\right) \cong 1.053$ (two equal tangent spheres), $S(\pi / 2)=1$ (sphere), and $S(\pi)=\pi^{2} / 9 \cong 1.097$ (circular disk). We wish to prove that $S(\alpha) \geq 1$ for $0 \leq \alpha \leq \pi$. We write

$$
S(\alpha)=\frac{\alpha^{2} g(\alpha)+G(\alpha)}{3 g^{3}(\alpha)}
$$

where

$$
g(\alpha)=\alpha \int_{-\infty}^{\infty}(1-\operatorname{th} \pi q \operatorname{th} \alpha q) d q=\int_{-\infty}^{\infty}(1-\operatorname{th} \pi q / \alpha \operatorname{th} q) d q
$$

and

$$
G(\alpha)=8 \alpha^{3} \int_{-\infty}^{\infty} \frac{q^{2} \operatorname{th} \pi q}{\operatorname{sh} 2 \alpha q} d q=8 \int_{-\infty}^{\infty} \frac{q^{2} \text { th } \pi q / \alpha}{\operatorname{sh} 2 q} d q
$$

We note that $g(\alpha)$ in (61) is the same function $g(\alpha)$ that was used in [3]. Next we let

$$
d(\alpha)=\alpha^{2} g(\alpha)+G(\alpha)
$$

so that

$$
S(\alpha)-1=\frac{d(\alpha)-3 g^{3}(\alpha)}{3 g^{3}(\alpha)} .
$$

Since $g(\alpha)>0$ for $0<\alpha \leq \pi$, it suffices to prove $d(\alpha)-3 g^{3}(\alpha) \geq 0$ in order to establish the inequality (2).

We note that $d(\alpha)-3 g^{3}(\alpha)$ has the value zero when $\alpha=\pi / 2$, because $S(\pi / 2)=1$. Its value when $\alpha=\pi / 4$ or $\pi$ can also be calculated as we shall see. In proving the desired inequality we shall find it convenient to estimate $d(\alpha)$ and $g(\alpha)$ by means of Taylor's series expansions of these functions in the neighborhoods of the points $\alpha=\pi / 4, \pi / 2$ and $\pi$. We shall therefore need to compute some of the derivatives of $g(\alpha), G(\alpha)$, and $d(\alpha)$, and to study their behavior.

From [3] we find that

$$
g^{\prime}(\alpha)=\pi \int_{-\infty}^{\infty} \frac{q \text { th } \alpha q}{\operatorname{ch}^{2} \pi q} d q
$$


(66)

$$
\begin{aligned}
& g^{\prime \prime}(a)=\pi \int_{-\infty}^{\infty} \frac{q^{2}}{\operatorname{ch}^{2} \pi q \operatorname{ch}^{2} a q} d q, \\
& g^{\prime \prime \prime}(a)=-2 \pi \int_{-\infty}^{\infty} \frac{q^{2} \operatorname{sh} c_{i} q}{\operatorname{ch}^{2} \pi q \operatorname{ch}^{3} \alpha_{q}} d q .
\end{aligned}
$$

It is clear that $g^{\prime}\left(\alpha_{\alpha}\right) \geq 0, g^{\prime \prime}(\alpha) \geq 0$ and $g^{\prime \prime \prime}\left(\alpha_{0}\right) \leq 0$, so that $g(\alpha)$ and $g^{\prime}\left(\alpha_{2}\right)$ are monotone increasing functions and $g^{\prime \prime}(\alpha)$ is a monotone decreasing function. Turning to the consideration of the derivatives of $G(u)$, we have

(68) $G^{\prime}\left(a_{2}\right)=-8 \pi a^{-2} \int_{-\infty}^{\infty} \frac{q^{2}}{\operatorname{sh} 2 q \operatorname{ch}^{2} \pi q / \alpha} d q=-8 \pi \alpha^{2} \int_{-\infty}^{\infty} \frac{q^{3}}{\operatorname{ch}^{2} \pi q \operatorname{sh} 2 \alpha q} d q$,

(69) $G^{\prime \prime}\left(\alpha_{i}\right)=2 \alpha^{-1} G^{\prime \prime}\left(\alpha_{i}\right)+16 \pi \alpha^{2} \int_{-\infty}^{\infty} \frac{q^{4} \operatorname{ch} 2 \alpha q}{\operatorname{ch}^{2} \pi q \operatorname{sh}^{2} 2 \alpha q} d q$,

$(70) G^{\prime \prime \prime}(\alpha)=-16 \pi \int_{-\infty}^{\infty} \frac{q^{3}}{\operatorname{ch}^{2} \pi q \operatorname{sh} 2 \alpha_{2} q} d q+64 \pi \alpha \int_{-\infty}^{\infty} \frac{q^{4} \operatorname{ch} 2 \alpha q}{\operatorname{ch}^{2} \pi q \operatorname{sh}^{2} 2 \alpha q} d q$

$$
-32 \pi \alpha^{2} \int_{-\infty}^{\infty} \frac{q^{5}\left(2+\operatorname{sh}^{2} 2 \alpha q\right)}{\operatorname{ch}^{2} \pi q \operatorname{sh}^{3} 2 a q} d q
$$

For the derivatives of $d\left(\mathcal{U}_{6}\right)$, we have

$$
\begin{aligned}
& d^{\prime}(a)=a_{2}^{2} g^{\prime}\left(a_{i}\right)+2 a g\left(a_{0}\right)+c_{i}^{\prime}\left(a_{i}\right), \\
& d^{\prime \prime}\left(a_{i}\right)=a^{2} g^{\prime \prime}\left(a_{1}\right)+4 a g^{\prime}\left(a_{i}\right)+2 g\left(a_{i}\right)+u^{\prime \prime}\left(a_{i}\right), \\
& d^{\prime \prime \prime}\left(a_{i}\right)=a_{2}^{2} g^{\prime \prime \prime}\left(a_{i}\right)+6 a_{0} g^{\prime \prime}\left(a_{i}\right)+6 g^{\prime}\left(a_{)}\right)+G^{\prime \prime \prime}\left(a_{i}\right) .
\end{aligned}
$$

We first consider the interval $\pi / 2 \leq \alpha \leq \pi$. If we let $\delta=\alpha-\pi / 2$, we have

$$
g(\alpha) \leq g(\pi / 2)+\delta g^{\prime}(\pi / 2)+\frac{\delta^{2}}{2} g^{\prime \prime}(\pi / 2), \quad \pi / 2 \leq \alpha \leq \pi,
$$

and

$$
d\left(\alpha_{i}\right) \geq d(\pi / 2)+\delta d^{\prime}(\pi / 2)+\frac{\delta^{2}}{2} d^{\prime \prime}(\pi / 2), \quad \pi / 2 \leq \alpha \leq \pi,
$$

since $g^{\prime \prime \prime}(\alpha) \leq 0$ by $(67)$, and $d^{\prime \prime \prime}(\alpha)>0$ for $\pi / 2 \leq \alpha \leq \pi$, as we now shall show. 
From (73) we find at once that

$$
d^{\prime \prime \prime}(\alpha) \geq \alpha^{2} g^{\prime \prime \prime}(\alpha)+6 \alpha g^{\prime \prime}(\pi)+6 g^{\prime}(\pi / 2)+G^{\prime \prime \prime}(\alpha), \quad \pi / 2 \leq \alpha \leq \pi .
$$

We must now find estimates for $g^{\prime \prime \prime}(\alpha)$ and $G^{\prime \prime \prime}(\alpha)$. From (67) we see that, for $\pi / 2 \leq \alpha \leq \pi$, we have

$$
\begin{aligned}
0 \leq-g^{\prime \prime \prime}(\alpha) & \leq 2 \pi \int_{-\infty}^{\infty} \frac{q^{3}}{\operatorname{ch}^{2} \pi q} \cdot \frac{\operatorname{sh} \pi q}{\operatorname{ch} \pi q} \cdot \frac{1}{\operatorname{ch}^{2} \pi q / 2} d q \\
& =32 \pi \int_{-\infty}^{\infty} \frac{q^{3} \operatorname{sh}^{2} \pi q(\operatorname{ch} \pi q-1)}{\operatorname{sh}^{3} 2 \pi q} d q \\
& =\pi \int_{-\infty}^{\infty} \frac{q^{3} \operatorname{sh} \pi q / 2}{\operatorname{sh}^{2} \pi q} d q-\pi \int_{-\infty}^{\infty} \frac{q^{3}(\operatorname{ch} \pi q-1)}{\operatorname{sh}^{3} \pi q} d q .
\end{aligned}
$$

If we make use of formulas (A-16), (A-32), and (A-31) in Appendix $A$ to evaluate these integrals, we find that

$$
0 \geq g^{\prime \prime \prime}(\alpha) \geq-1 / \pi+3-7 \pi / 8, \quad \pi / 2 \leq \alpha \leq \pi .
$$

Equation (70) shows that $G^{\prime \prime \prime}(\alpha)$ is the sum of three integrals each of which may be estimated by methods similar to that used above in the estimation of $g^{\prime \prime \prime}(\alpha)$; it is convenient to observe that the function $q / \mathrm{sh} q$ decreases monotonely for $q>0$ and is an even function of $q$. The necessary formulas from Appendix A are $(\mathrm{A}-16),(\mathrm{A}-18),(\mathrm{A}-20),(\mathrm{A}-26)$, and $(\mathrm{A}-30)$. We find that

$$
\begin{aligned}
& G^{\prime \prime \prime}(\alpha) \geq[\pi /(2 \alpha)]\left(12-44 \pi-25 \pi^{2}+12 \pi^{3}\right) \\
& \quad+34 \pi / 45-16 /(3 \pi)+\pi \alpha(25-8 \pi), \pi / 2 \leq \alpha \leq \pi .
\end{aligned}
$$

The values of $g^{\prime \prime}(\pi)$ and $g^{\prime}(\pi / 2)$ are given in equations (B-3) and (B-5) of Appendix B. If we substitute these values as well as values from (77) and (78) into (76), we find that, for $\pi / 2 \leq \alpha \leq \pi, \alpha d^{\prime \prime \prime}(\alpha)$ is not less than a certain polynomial of third degree in $\alpha$. It is easily verified that this polynomial has three real zeros, none of which lies between $\pi / 2$ and $\pi$, and that it is positive for $\alpha=\pi / 2$ and $\alpha=\pi$. Consequently, it is positive for $\pi / 2 \leq \alpha \leq \pi$. It follows that $d^{\prime \prime \prime}(\alpha)>0$ in the same interval.

If we substitute into (74) and (75) the necessary values from Appendix B, we find that, for $\pi / 2 \leq \alpha \leq \pi, \delta^{-2}\left[d(\alpha)-3 g^{3}(\alpha)\right]$ is not less than a certain fourth degree polynomial in $\delta$ which is readily shown to decrease monotonely as 
$\delta$ increases from 0 . Moreover, this polynomial is positive for $\delta=4 / 3$. It follows that

$$
d(\alpha)-3 g^{3}(\alpha) \geq 0, \quad \pi / 2 \leq \alpha \leq \pi / 2+\frac{4}{3} .
$$

Since the desired inequality has not yet been proven for $\alpha>\pi / 2+4 / 3$, we consider further the interval $\pi / 2 \leq \alpha \leq \pi$ and let $\epsilon=\pi-\alpha$. lie first recall that $g^{\prime \prime}(\alpha)$ decreases monotonely. Also it has been shown that $a^{\prime \prime \prime}\left(\alpha_{0}\right)>0$ for $\pi / 2 \leq$ $a \leq \pi$; it follows that $d^{\prime \prime}(\alpha)$ increases monotonely in this interval. We thus obtain

$$
g\left(a_{i}\right) \leq g(\pi)-\epsilon_{\xi^{\prime}}(\pi)+\frac{\epsilon^{2}}{2} g^{\prime \prime}(\pi / 2), \quad \pi / 2 \leq \alpha \leq \pi,
$$

and

$$
d(\alpha) \geq d(\pi)-\epsilon d^{\prime}(\pi)+\frac{\epsilon^{2}}{2} d^{\prime \prime}(\pi / 2), \quad \pi / 2 \leq \alpha \leq \pi .
$$

If we substitute into (80) and (81) the necessary values from Appendix $B$, we find that, for $\pi / 2 \leq c_{i} \leq \pi, d\left(c_{i}\right)-3 g^{3}\left(\alpha_{i}\right)$ is not less than a certain sixth degree polynomial in $\epsilon$ which is readily shown to be positive for $0 \leq \epsilon \leq 1 / 2$. It follows that

$$
d(a)-3 g^{3}(\alpha)>0, \quad \pi-\frac{1}{2} \leq \alpha \leq \pi .
$$

If we conbine (79) and (82) the desired inequality is proven for the interval $\pi / 2 \leq$ $u \leq \pi$.

Next we turn our attention to the interval $\pi / 4 \leq \dot{u} \leq \pi / 2$. We first need to obtain estimates for $g^{\prime \prime \prime}(\alpha)$ and $d^{\prime \prime \prime}\left(\alpha_{0}\right)$ in this interval. If we make use of (67) and eniploy $(A-16)$ and $(A-28)$ in Appendix $A$ to evaluate the integrals which arise, we find that

$$
0 \geq g^{\prime \prime \prime}(\alpha) \geq-\frac{3}{2}+7 \pi / 16, \quad 0 \leq \alpha \leq \pi / 2 .
$$

Before we can estimate $d^{\prime \prime \prime}(\alpha)$ we need to estimate $G^{\prime \prime \prime}(\alpha)$. We proceed as we did for the interval $\pi / 2 \leq \alpha \leq \pi$, and we find that two of the integrals that have to be evaluated are the same as before although the inequalities are reversed. llowever, the third integral is different; it may be estimated by making use of (A-8), (A-10), and (A-17). We find that 
(84)

$$
\begin{aligned}
G^{\prime \prime \prime}(\alpha) \leq & {[\pi /(2 \alpha)]\left(12-44 \pi-25 \pi^{2}+12 \pi^{3}\right)+512 \pi } \\
& +176 \pi(2)^{1 / 2}-171 \pi^{2}(2)^{1 / 2}+\pi \alpha(25-8 \pi), \quad \pi / 4 \leq \alpha \leq \pi / 2 .
\end{aligned}
$$

If we recall that $g^{\prime}(\alpha)$ is an increasing function, $g^{\prime \prime}(\alpha)$ a decreasing function, and $g^{\prime \prime \prime}(\alpha)$ a nonpositive function, we find from (73) that

$$
d^{\prime \prime \prime}(\alpha) \leq 0+6 a g^{\prime \prime}(0)+6 g^{\prime}(\pi / 2)+G^{\prime \prime \prime}(\alpha), \quad \pi / 4 \leq \alpha \leq \pi / 2 .
$$

If we insert the values of $g^{\prime \prime}(0)$ and $g^{\prime}(\pi / 2)$ from Appendix $B$, and make use of (84), we obtain

$$
\begin{gathered}
d^{\prime \prime \prime}(a) \leq[\pi /(2 a)]\left(12-44 \pi-25 \pi^{2}+12 \pi^{3}\right)+6+1021 \pi / 2 \\
+176 \pi(2)^{1 / 2}-171 \pi^{2}(2)^{1 / 2}+a\left(1+25 \pi-8 \pi^{2}\right) \\
\leq 18+467 \pi+176 \pi(2)^{1 / 2}-25 \pi^{2} / 2 \\
-171 \pi^{2}(2)^{1 / 2}+8 \pi^{3}, \quad \pi / 4 \leq a \leq \pi / 2 .
\end{gathered}
$$

In passing to the last inequality we have replaced $\alpha_{0}$ by $\pi / 2$ because the first parenthesis is negative and the last one is positive. We also point out that the last member of (85) is positive.

If we now let $\zeta=\pi / 2-\alpha$, we find from the Taylor's series expansions of $g(\alpha)$ and $d(\alpha)$, on using (83) and (85), that

$$
\begin{aligned}
g(a) \leq g(\pi / 2)-\zeta g^{\prime} & (\pi / 2)+\frac{\zeta^{2}}{2} g^{\prime \prime}(\pi / 2) \\
& +\zeta^{3}\left(\frac{1}{4}-7 \pi / 96\right), \quad \pi / 4 \leq \alpha \leq \pi / 2
\end{aligned}
$$

and

$$
d(\alpha) \geq d(\pi / 2)-\zeta d^{\prime}(\pi / 2)+\frac{\zeta^{2}}{2} d^{\prime \prime}(\pi / 2)-\frac{\zeta^{3}}{6} d_{1}, \quad \pi / 4 \leq \alpha<\pi / 2,
$$

where $d_{1}$ denotes the last member of (85).

If we substitute into (86) and (87) the necessary values from Appendix B, we find that, for $\pi / 4 \leq \alpha \leq \pi / 2, \zeta^{-2}\left[d(\alpha)-3 g^{3}(\alpha)\right]$ is not less than a certain seventh degree polynomial in $\zeta$ which is easily shown to be positive for $0 \leq \zeta$ $\leq 1 / 2$. It follows that

$$
d(\alpha)-3 g^{3}(\alpha) \geq 0, \quad \pi / 2-\frac{1}{2} \leq \alpha \leq \pi / 2 .
$$


Since the desired inequality has not yet been proven for $\alpha<\pi / 2-1 / 2$, we consider further the interval $\pi / 4 \leq \alpha \leq \pi / 2$ and let $\eta=\alpha-\pi / 4$. We need another estimate for $d^{\prime \prime \prime}(\alpha)$, but of the opposite sense to that given by (85). This in turn requires a new estimate for $G^{\prime \prime \prime}(\alpha)$. The necessary integrals may be evaluated by using $(A-17),(A-23)$, and $(A-24)$. We find that

$$
G^{\prime \prime \prime}(\alpha) \geq-8 /(3 \alpha)+32 \pi-10 \pi^{2}-14 \alpha / 15, \quad 0<a \leq \pi / 2 .
$$

From (73) we find at once that

$$
d^{\prime \prime \prime}(\alpha) \geq \alpha^{2} g^{\prime \prime}(\alpha)+6 \alpha g^{\prime \prime}(\pi / 2)+6 g^{\prime}(\pi / 4)+G^{\prime \prime \prime}(\alpha), \quad \pi / 4 \leq \alpha \leq \pi / 2 .
$$

If we insert the necessary values from Appendix B, and make use of (83) and (89), we obtain

$$
\begin{aligned}
d^{\prime \prime \prime}(\alpha) \geq & \frac{1}{a^{\prime}}\left\{-8 / 3+\left[12(2)^{1 / 2}-6+3 \pi(2)^{1 / 2}+49 \pi / 2-10 \pi^{2}\right] \alpha\right. \\
& \left.+(136 / 15-3 \pi) \alpha^{2}+(7 \pi / 16-3 / 2) \alpha^{3}\right\}, \quad \pi / 4 \leq \alpha \leq \pi / 2 .
\end{aligned}
$$

But it is easily shown that the polynomial in the braces increases monotonely when $\alpha$ increases from 0 to $\pi / 2$. Moreover, it is negative if $\alpha=\pi / 4$. Hence we may replace $\alpha$ by $\pi / 4$ in the right-hand member of (90). If we denote the resulting value by $d_{2}$, we see that $d^{\prime \prime \prime}\left(\alpha_{2}\right) \geq d_{2}$ for $\pi / 4 \leq \alpha \leq \pi / 2$. Using this fact and recalling that $g^{\prime \prime \prime}(\alpha) \leq 0$, we have

$$
g(\alpha) \leq g(\pi / 4)+\eta g^{\prime}(\pi / 4)+\frac{\eta^{2}}{2} g^{\prime \prime}(\pi / 4), \quad \pi / 4 \leq \alpha \leq \pi / 2,
$$

and

$$
d(\alpha) \geq d(\pi / 4)+\eta d^{\prime}(\pi / 4)+\frac{\eta^{2}}{2} d^{\prime \prime}(\pi / 4)+\frac{\eta^{3}}{6} d_{2}, \pi / 4 \leq \alpha \leq \pi / 2 .
$$

If we substitute into (91) and (92) the necessary values from Appendix B, we find that, for $\pi / 4 \leq \alpha \leq \pi / 2, d(\alpha)-3 g^{3}(\alpha)$ is not less than a certain sixth degree polynomial in $\eta$ which is easily shown to be positive for $0 \leq \eta \leq 0.4$. It follows that

$$
d(\alpha)-3 g^{3}(\alpha)>0, \quad \pi / 4 \leq \alpha \leq \pi / 4+0.4 .
$$


If we combine (88) and (93), the desired inequality is proven for the interval $\pi / 4$ $\leq u \leq \pi / 2$.

Finally we consider the interval $0 \leq \alpha \leq \pi / 4$. We first need to obtain estimates for $d^{\prime \prime}(\alpha)$ and $G^{\prime \prime}(\alpha)$ in this interval. If we make use of (68) and (69), and employ $(A-22),(A-23),(A-24)$, and $(A-30)$ to evaluate the integrals which arise, we find that

$$
G^{\prime \prime}(a) \geq-4 / 3+121 \pi^{2} / 60-5 \pi^{3} / 8, \quad 0 \leq 0 \leq \pi / 4 .
$$

From (72) we find at once that

$$
d^{\prime \prime}(\alpha) \geq 2 g(0)+G^{\prime \prime}(\alpha), \quad 0 \leq \alpha \leq \pi / 4,
$$

since $g^{\prime \prime}(\alpha)$ and $g^{\prime}(\alpha)$ are both nonnegative. If we take the value of $g(0)$ from Appendix B, and make use of (94), we find that

$$
d^{\prime \prime}(\alpha) \geq 4 \log 2-4 / 3+121 \pi^{2} / 60-5 \pi^{3} / 8, \quad 0 \leq a \leq \pi / 4 .
$$

If we now let $k=\pi / 4-\alpha_{2}$, recall that $g^{\prime \prime}\left(\alpha_{0}\right)$ decreases monotonely when $\alpha$ increases, and make use of $(95)$, we find that

$$
g(\alpha) \leq g(\pi / 4)-\kappa^{\prime}(\pi / 4)+\frac{k^{2}}{2} g^{\prime \prime}(0), \quad 0 \leq \alpha \leq \pi / 4,
$$

and

$$
d(\alpha) \geq d(\pi / 4)-\kappa d^{\prime}(\pi / 4)+\frac{\kappa^{2}}{2} d_{3}, \quad 0 \leq \alpha \leq \pi / 4,
$$

where $d_{3}$ denotes the right-hand member of (95).

If we substitute into (96) and (97) the necessary values from Appendix 13, we find that, for $0 \leq \alpha \leq \pi / 4, d(\alpha)-3 g^{3}(\alpha)$ is not less than a certain sixth degree polynomial in $k$ which is positive for $0 \leq \kappa \leq \pi / 4$. It follows that

$$
d(\alpha)-3 g^{3}(\alpha)>0, \quad 0 \leq a \leq \pi / 4 .
$$

Combining this with our previous results, we see that the desired inequality has now been established for the whole interval $0 \leq \alpha \leq \pi$. As previously observed, this proves the inequality (2) for the symmetric lens.

7. Appendix A. In this appendix we give a table of integrals which includes 
all the integrals needed in the proof in $\$ 6$ and in the calculations in Appendix B. Some of these integrals can be deduced easily from formulas given in the integral tables of Bierens de Haan [1]. When this is the case the formula is followed by two numbers in parenthesis giving first the table number and second the formula number of the necessary formula in the tables of Bierens de Haan.

Since not all of our formulas can be deduced from these tables we indicate alternative methods of proof. Formulas $(A-1)$ to $(A-6)$ can be derived by standard methods of contour integration. In connection with $(A-5)$, we mention that it is necessary to integrate both $z$ sh $\mathcal{u}^{\prime} z / \mathrm{sh}^{2} \pi z$ and $\operatorname{ch} \alpha z / \operatorname{sh}^{2} \pi z$ around an indented rectangular contour; and in (A-6) it is necessary to integrate $z^{2} \operatorname{sh} \alpha z / \operatorname{sh}^{3} \pi z$, $z \operatorname{ch} \alpha z / \operatorname{sh}^{3} \pi z$, and $\operatorname{sh} \alpha z / \mathrm{sh}^{3} \pi z$ around the same contour. Forniulas (A-7) to $(A-20)$ can be derived by differentiation of the formulas $(A-1)$ to $(A-6)$. Finally, formulas $(A-21)$ to $(A-32)$ are all special or limiting cases of formulas $(A-7)$ to $(A-20)$. It may be noted that $(A-32)$ may be derived most easily by using an integration by parts and $(\Lambda-29)$.

$$
\begin{aligned}
& (\mathrm{A}-1) \quad \int_{-\infty}^{\infty} \frac{\operatorname{ch} \alpha q}{\operatorname{ch} \pi q} d q=\sec \frac{\alpha}{2}, \quad-\pi<\alpha<\pi \\
& \text { (A-2) } \int_{-\infty}^{\infty} \frac{\operatorname{ch} \alpha q}{\operatorname{ch}^{2} \pi q} d q=\frac{\alpha}{\pi} \csc \frac{a}{2}, \quad-2 \pi<\alpha<2 \pi \\
& \text { (A-3) } \int_{-\infty}^{\infty} \frac{\operatorname{ch} \alpha q}{\operatorname{ch}^{4} \pi q} d q=\frac{\alpha}{6 \pi^{3}}\left(4 \pi^{2}-\alpha^{2}\right) \csc \frac{\alpha}{2}, \quad-4 \pi<\alpha<4 \pi \\
& (A-4) \quad \int_{-\infty}^{\infty} \frac{\operatorname{sh} \alpha q}{\operatorname{sh} \pi q} d q=\tan \frac{\alpha}{2}, \quad-\pi<\alpha<\pi \\
& (27,10) \\
& (A-5) \int_{-\infty}^{\infty} \frac{q \operatorname{sh} \alpha q}{\operatorname{sh}^{2} \pi q} d q=\frac{1}{2 \pi}\left(\alpha \csc ^{2} \frac{\alpha}{2}-2 \cot \frac{\alpha}{2}\right), \quad-2 \pi<\alpha<2 \pi \\
& \text { (A-6) } \int_{-\infty}^{\infty} \frac{q^{2} \operatorname{sh} c q}{\operatorname{sh}^{3} \pi q} d q=\frac{1}{4 \pi^{2}}\left[4 \tan \frac{\alpha}{2}+4 \alpha \sec ^{2} \frac{\alpha}{2}\right. \\
& \left.+\left(\alpha^{2}-\pi^{2}\right) \sec ^{2} \frac{\hat{\alpha}}{2} \tan \frac{\alpha}{2}\right], \quad-3 \pi<\alpha<3 \pi
\end{aligned}
$$


(A-7) $\quad \int_{-\infty}^{\infty} \frac{q^{2} \operatorname{ch} a q}{\operatorname{ch} \pi q} d q=\frac{1}{4} \sec \frac{\alpha}{2}\left(1+2 \tan ^{2} \frac{\alpha}{2}\right), \quad-\pi<\alpha<\pi$

(A-8) $\int_{-\infty}^{\infty} \frac{q^{4} \operatorname{ch~} c_{q}}{\operatorname{ch} \pi q} d q=\frac{1}{16} \sec \frac{c_{2}}{2}\left(5+28 \tan ^{2} \frac{a}{2}+24 \tan ^{4} \frac{a}{2}\right)$,

$$
-\pi<a<\pi \quad(82,16)
$$

(A-9) $\int_{-\infty}^{\infty} \frac{q^{2} \operatorname{ch} a q}{\operatorname{ch}^{2} \pi q} d q=\frac{1}{4 \pi} \csc \frac{c_{i}}{2}\left[-4 \cot \frac{a}{2}\right.$

$$
\left.+a\left(1+2 \cot ^{2} \frac{\alpha}{2}\right)\right], \quad-2 \pi<\alpha<2 \pi
$$

(4-10) $\int_{-\infty}^{\infty} \frac{q^{4} \operatorname{ch} \alpha q}{\operatorname{ch}^{2} \pi q} d q=\frac{1}{16 \pi} \csc \frac{\alpha}{2}\left[-8 \cot \frac{\alpha}{2}\left(5+6 \cot ^{2} \frac{\alpha}{2}\right)\right.$

$$
\left.+\alpha\left(5+28 \cot ^{2} \frac{\alpha}{2}+24 \cot ^{4} \frac{\alpha}{2}\right)\right], \quad-2 \pi<\alpha<2 \pi
$$

(A-11) $\int_{-\infty}^{\infty} \frac{q^{2} \operatorname{ch} \alpha q}{\operatorname{ch}^{4} \pi q} d q=\frac{1}{24 \pi^{3}} \csc \frac{\alpha}{2}\left[-24 \alpha+4\left(3 \alpha^{2}-4 \pi^{2}\right) \cot \frac{\alpha}{2}\right.$

$$
\left.+\alpha\left(4 \pi^{2}-\alpha^{2}\right)\left(1+2 \cot ^{2} \frac{\alpha}{2}\right)\right], \quad-4 \pi<\alpha<4 \pi
$$

(A-12) $\int_{-\infty}^{\infty} \frac{q^{4} \operatorname{ch} \alpha q}{\operatorname{ch}^{4} \pi q} d q=\frac{1}{96 \pi^{3}} \csc \frac{\alpha}{2}\left[192 \cot \frac{\alpha}{2}-144 \alpha\left(1+2 \cot ^{2} \frac{\alpha}{2}\right)\right.$

$$
\begin{aligned}
& +8\left(3 a^{2}-4 \pi^{2}\right) \cot \frac{\alpha}{2}\left(5+6 \cot ^{2} \frac{\alpha}{2}\right) \\
& +\alpha\left(4 \pi^{2}-\alpha^{2}\right)\left(5+28 \cot ^{2} \frac{\alpha}{2}\right. \\
& \left.\left.+24 \cot ^{4} \frac{\alpha}{2}\right)\right], \quad-4 \pi<\alpha<4 \pi
\end{aligned}
$$

$(\mathrm{A}-13) \quad \int_{-\infty}^{\infty} \frac{q \operatorname{ch} \alpha q}{\operatorname{sh} \pi q} d q=\frac{1}{2} \sec ^{2} \frac{\alpha}{2}, \quad-\pi<\alpha<\pi$ 
$(\lambda-14) \quad \int_{-\infty}^{\infty} \frac{q^{3} \operatorname{ch} \alpha q}{\operatorname{sh} \pi q} d q=\frac{1}{4} \sec ^{2} \frac{a}{2}\left(1+3 \tan ^{2} \frac{\alpha}{2}\right), \quad-\pi<a<\pi \quad(82,15)$

$(A-15) \quad \int_{-\infty}^{\infty} \frac{q^{2} \operatorname{ch} \alpha q}{\operatorname{sh}^{2} \pi q} d q=\frac{1}{2 \pi} \csc ^{2} \frac{\alpha}{2}\left(2-\alpha \cot \frac{\alpha}{2}\right), \quad-2 \pi<\alpha<2 \pi$

$(1-16) \quad \int_{-\infty}^{\infty} \frac{q^{3} \operatorname{sh} \alpha q}{\operatorname{sh}^{2} \pi q} d q=\frac{1}{4 \pi} \csc ^{2} \frac{\alpha}{2}\left[-6 \cot \frac{\alpha}{2}\right.$

$$
\left.+a\left(1+3 \cot ^{2} \frac{a}{2}\right)\right], \quad-2 \pi<a<2 \pi
$$

(i-17) $\int_{-\infty}^{\infty} \frac{q^{4} \operatorname{ch} \alpha q}{\operatorname{sh}^{2} \pi q} d q=\frac{1}{2 \pi} \csc ^{2} \frac{\alpha}{2}\left[2\left(1+3 \cot ^{2} \frac{\alpha}{2}\right)\right.$

$$
\left.-a \cot \frac{\omega}{2}\left(2+3 \cot ^{2} \frac{\omega_{0}}{2}\right)\right], \quad-2 \pi<\omega_{0}<2 \pi
$$

$(A-18) \quad \int_{-\infty}^{\infty} \frac{q^{5} \operatorname{sh} a q}{\operatorname{sh}^{2} \pi q} d q=\frac{1}{4 \pi} \csc ^{2} \frac{a}{2}\left[-10 \cot \frac{a}{2}\left(2+3 \cot ^{2} \frac{a}{2}\right)\right.$

$$
\left.+\alpha\left(2+15 \cot ^{2} \frac{\alpha}{2}+15 \cot ^{4} \frac{\alpha}{2}\right)\right], \quad-2 \pi<\alpha<2 \pi
$$

$(1-19) \quad \int_{-\infty}^{\infty} \frac{q^{3} \operatorname{ch} \alpha q}{\operatorname{sh}^{3} \pi q} d q=\frac{1}{8 \pi^{2}} \sec ^{2} \frac{a^{2}}{2}\left[12+12 \alpha \tan \frac{\alpha}{2}\right.$

$$
\left.+\left(\alpha^{2}-\pi^{2}\right)\left(1+3 \tan ^{2} \frac{\alpha}{2}\right)\right],-3 \pi<\alpha<3 \pi
$$

$(A-20) \quad \int_{-\infty}^{\infty} \frac{q^{5} \operatorname{ch} a q}{\operatorname{sh}^{3} \pi q} d q=\frac{1}{8 \pi^{2}} \sec ^{2} \frac{a_{2}}{2}\left[20\left(1+3 \tan ^{2} \frac{a}{2}\right)\right.$

$$
\begin{gathered}
+20 \alpha \tan \frac{a}{2}\left(2+3 \tan ^{2} \frac{u}{2}\right) \\
\left.+\left(\alpha^{2}-\pi^{2}\right)\left(2+15 \tan ^{2} \frac{a}{2}+15 \tan ^{4} \frac{\alpha}{2}\right)\right],-3 \pi<\alpha<3 \pi
\end{gathered}
$$


$(A-21)$

$$
\int_{-\infty}^{\infty} \frac{q^{2}}{\operatorname{ch} \pi q} d q=\frac{1}{4} \quad(84,3)
$$

$$
\int_{-\infty}^{\infty} \frac{q^{4}}{\operatorname{ch} \pi q} d q=\frac{5}{16} \quad(84,7)
$$

$$
\int_{-\infty}^{\infty} \frac{q^{2}}{\operatorname{ch}^{2} \pi q} d q=\frac{1}{6 \pi} \quad(86,2)
$$

$$
\int_{-\infty}^{\infty} \frac{q^{4}}{\operatorname{ch}^{2} \pi q} d q=\frac{7}{120 \pi} \quad(86,2)
$$

$$
\int_{-\infty}^{\infty} \frac{q^{2}}{\operatorname{ch}^{4} \pi q} d q=\frac{1}{9 \pi}-\frac{2}{3 \pi^{3}}
$$

$$
\int_{-\infty}^{\infty} \frac{q^{4}}{\operatorname{ch}^{4} \pi q} d q=\frac{7}{180 \pi}-\frac{1}{3 \pi^{3}}
$$

$$
\int_{-\infty}^{\infty} \frac{q}{\operatorname{sh} \pi q} d q=\frac{1}{2} \quad(84,2)
$$

$$
\int_{-\infty}^{\infty} \frac{q^{3}}{\operatorname{sh} \pi q} d q=\frac{1}{4} \quad(84,5)
$$

$$
\int_{-\infty}^{\infty} \frac{q^{2}}{\operatorname{sh}^{2} \pi q} d q=\frac{1}{3 \pi} \quad(86,5)
$$

$$
\int_{-\infty}^{\infty} \frac{q^{4}}{\operatorname{sh}^{2} \pi q} d q=\frac{1}{15 \pi} \quad(86, \tilde{\jmath})
$$

$$
\int_{-\infty}^{\infty} \frac{q^{3}}{\operatorname{sh}^{3} \pi q} d q=\frac{3}{2 \pi^{2}}-\frac{1}{8}
$$

$$
\int_{-\infty}^{\infty} \frac{q^{3} \operatorname{ch} \pi q}{\operatorname{sh}^{3} \pi q} d q=\frac{1}{2 \pi^{2}}
$$

8. Appendix B. In the proof given in $\$ 6$ we had to use the values of $g(\alpha)$ and $d(\alpha)$ for certain values of $\alpha$. The necessary values are listed in this appendix; 
the method of calculation of each is also indicated. Following is the list of values used:

$$
\begin{array}{ll}
(\mathrm{B}-1,2,3) & g(\pi)=2, \quad g^{\prime}(\pi)=1 / \pi, \quad g^{\prime \prime}(\pi)=1 / 9-2 /\left(3 \pi^{2}\right) \\
(\mathrm{B}-4,5,6) & g(\pi / 2)=\pi / 2, \quad g^{\prime}(\pi / 2)=1-\pi / 4, \quad g^{\prime \prime}(\pi / 2)=5 / 3-\pi / 2 \\
(\mathrm{~B}-7) & g(\pi / 4)=\pi(2)^{1 / 2} / 2-\pi / 4 \\
(\mathrm{~B}-8) & g^{\prime}(\pi / 4)=-1+2(2)^{1 / 2}-5 \pi / 4+\pi(2)^{1 / 2} / 2 \\
(\mathrm{~B}-9) & g^{\prime \prime}(\pi / 4)=34 / 3+4(2)^{1 / 2}+\pi-9 \pi(2)^{1 / 2} / 2 \\
(\mathrm{~B}-10,11) & g(0)=2 \log 2, \quad g^{\prime \prime}(0)=1 / 6 \\
(\mathrm{~B}-12,13) & d(\pi)=8 \pi^{2} / 3, \quad d^{\prime}(\pi)=6 \pi-\pi^{3} / 8 \\
(\mathrm{~B}-14,15) & d(\pi / 2)=3 \pi^{3} / 8, \quad d^{\prime}(\pi / 2)=9 \pi^{2} / 4-9 \pi^{3} / 16 \\
(\mathrm{~B}-16) & d^{\prime \prime}(\pi / 2)=9 \pi^{2}+23 \pi^{2} / 12-11 \pi^{3} / 8 \\
(\mathrm{~B}-17) & d(\pi / 4)=7 \pi^{3}(2)^{1 / 2} / 32-\pi^{3} / 64 \\
(\mathrm{~B}-18) & d^{\prime}(\pi / 4)=-3 \pi^{2} / 16+21 \pi^{2}(2)^{1 / 2} / 8-133 \pi^{3} / 64+23 \pi^{3}(2)^{1 / 2} / 32 \\
(\mathrm{~B}-19) & d^{\prime \prime}(\pi / 4)=-3 \pi / 2+21 \pi^{1 / 2}(2)^{1 / 2}+371 \pi^{2} / 24+69 \pi^{2}(2)^{1 / 2} / 4 \\
&
\end{array}
$$

Formulas (B-4), (B-5), (B-6), (B-10), and (B-11) will be found in $[3] ;(B-1)$ and (B-2) can be proven by starting with (61) and (65) and using (A-2), an integration by parts being first needed in the case of (B-2). (B-3) follows at once from (66) and (A-25). In order to prove (B-7) we observe that

$$
g(\pi / 4)=\frac{\pi}{4} \int_{-\infty}^{\infty} \frac{\operatorname{ch} 3 \pi q / 4}{\operatorname{ch} \pi q \operatorname{ch} \pi q / 4} d q=\frac{\pi}{4} \int_{-\infty}^{\infty} \frac{2 \operatorname{ch} \pi q / 2-1}{\operatorname{ch} \pi q} d q,
$$

and the result follows from (A-1). For (B-8) we have

$$
\zeta^{\prime}(\pi / 4)=\pi \int_{-\infty}^{\infty} \frac{q \operatorname{sh} \pi q / 4}{\operatorname{ch}^{2} \pi q \operatorname{ch} \pi q / 4} d q=\pi \int_{-\infty}^{\infty} \frac{q(\operatorname{ch} \pi q+1-2 \operatorname{ch} \pi q / 2)}{\operatorname{ch}^{2} \pi q \operatorname{sh} \pi q} d q
$$




$$
=2 \pi \int_{-\infty}^{\infty} q\left[\frac{1}{\operatorname{sh} 2 \pi q}+\frac{2(\operatorname{sh} \pi q-\operatorname{sh} 3 \pi q / 2-\operatorname{sh} \pi q / 2)}{\operatorname{sh}^{2} 2 \pi q}\right] d q,
$$

and the result follows from $(A-27)$ and $(\Lambda-5)$. For (B-9) we start with (66) and have

$$
\begin{aligned}
g^{\prime \prime}(\pi / 4) & =4 \pi \int_{-\infty}^{\infty} \frac{q^{2} \operatorname{sh}^{2} \pi q / 4}{\operatorname{ch}^{2} \pi q \operatorname{sh}^{2} \pi q / 2} d q \\
& =16 \pi \int_{-\infty}^{\infty} \frac{q^{2}(\operatorname{ch} \pi q / 2-1)(\operatorname{ch} \pi q+1)}{\operatorname{sh}^{2} 2 \pi q} d q \\
& =\pi \int_{-\infty}^{\infty} \frac{q^{2}(\operatorname{ch} 3 \pi q / 4-2 \operatorname{ch} \pi q / 2+3 \operatorname{ch} \pi q / 4-2)}{\operatorname{sh}^{2} \pi q} d q,
\end{aligned}
$$

and the result follows from $(\lambda-15)$ and $(A-29)$.

Formulas (B-12) to (B-19) follow immediately from (63), (71), and (72) if we show how to calculate the following:

$(B-20,21) \quad G(\pi)=2 \pi^{2} / 3, \quad G^{\prime}(\pi)=\pi-\pi^{3} / 8$

$(13-22,23) \quad G(\pi / 2)=\pi^{3} / 4, \quad G^{\prime}(\pi / 2)=3 \pi^{3} / 2-\pi^{4} / 2$

$(B-24) \quad G^{\prime \prime}(\pi / 2)=6 \pi+2 \pi^{2}-5 \pi^{3} / 4$

(B-25) $\quad G(\pi / 4)=3 \pi^{3}(2)^{1 / 2} / 16$

(B-26) $\quad G^{\prime}(\pi / 4)=9 \pi^{2}(2)^{1 / 2} / 4-2 \pi^{3}+11 \pi^{3}(2)^{1 / 2} / 16$

$(\mathrm{B}-27) \quad G^{\prime \prime}(\pi / 4)=18 \pi(2)^{1 / 2}+16 \pi^{2}+33 \pi^{2}(2)^{1 / 2} / 2-171 \pi^{3}(2)^{1 / 2} / 16$

Formula (B3-20) follows easily from (62) and (A-23), (B-22) from (62) and (A-21), (B-23) from (68) and (A-16), (B-24) from (69) and (A-17), and (B-25) from (62) and $(\mathrm{A}-7)$. For $(\mathrm{B}-2 \mathrm{I})$ we use $(68)$ and find that

$$
G^{\prime}(\pi)=-8 \pi^{3} \int_{-\infty}^{\infty} \frac{q^{3}}{\operatorname{ch}^{2} \pi q \operatorname{sh} 2 \pi q} d q=-16 \pi^{3} \int_{-\infty}^{\infty} \frac{q^{3}(\operatorname{ch} 2 \pi q-1)}{\operatorname{sh}^{3} 2 \pi q} d q,
$$

whence the result follows from (A-32) and (A-31). For (B-26) we obtain, from (68), $G^{\prime}(\pi / 4)=-\pi^{3} \int_{-\infty}^{\infty} \frac{q^{3} \operatorname{ch} \pi q / 2}{\operatorname{ch}^{2} \pi q \operatorname{sh} \pi q} d q=-2 \pi^{3} \int_{-\infty}^{\infty} \frac{q^{3}(\operatorname{sh} 3 \pi q / 2+\operatorname{sh} \pi q / 2)}{\operatorname{sh}^{2} 2 \pi q} d q$, 
and the result follows from (A-16). Finally for ( $\$ 3-27)$ we obtain, from (69),

$$
\begin{aligned}
G^{\prime \prime}(\pi / 4)= & (8 / \pi) G^{\prime}(\pi / 4)+\pi^{3} \int_{-\infty}^{\infty} \frac{q^{4} \operatorname{ch} \pi q / 2}{\operatorname{ch}^{2} \pi q \operatorname{sh}^{2} \pi q / 2} d q \\
= & (8 / \pi) G^{\prime}(\pi / 4)+2 \pi^{3} \int_{-\infty}^{\infty} \frac{q^{4} \operatorname{ch} \pi q / 2}{\operatorname{ch}^{2} \pi q(\operatorname{ch} \pi q-1)} d q \\
= & (8 / \pi) G^{\prime}(\pi / 4) \\
& +2 \pi^{3} \int_{-\infty}^{\infty} q^{4} \operatorname{ch~} \pi q / 2\left(\frac{1}{2 \operatorname{sh}^{2} \pi q / 2}-\frac{1}{\operatorname{ch} \pi q}-\frac{1}{\operatorname{ch}^{2} \pi q}\right) d q,
\end{aligned}
$$

and the result follows from $(A-17),(A-8)$, and $(A-10)$.

\section{References}

1. D. Bierens de Haan, Nouvelles tables d'intégrales définies, G. E. Stechert, New York, 1939.

2. E. T. Copson, Functions of a complex variable, Oxford Liniversity Press, Oxford, 1935.

3. J. G. Herriot, Inequalities for the capacity of a lens, Duke Math. J. 15 (1948), 743-753.

4. G. Pólya and G. Szegö, Inequalities for the capacity of a condenser, Amer. J. Nath. 67 (1945), 1-32.

5. - Isoperimetric inequalities in mathematical physics, Annals of Mathematics Studies, Princeton Lniversity Press, Princeton, in print.

6. M. Schiffer and G. Szegö, Virtual mass and polarization, Trans. Amer. Math. Soc. 67 (1949), 130-205.

7. G. Szegö, On the capacity of a condenser, Bull. Amer. Math. Soc. 51 (1945), 325-350.

Stanford University 



\title{
PACIFIC JOURNAL OF MATHEMATICS
}

\section{EDITORS}

\author{
HERBERT BUSEMANN \\ University of Southern California \\ R. M. RoBINSON \\ Los Angeles 7, California \\ University of California \\ Berkeley 4, California \\ E. F. BECKENBACH, Managing Editor \\ University of California \\ Los Angeles 24, California
}

\section{ASSOCIATE EDITORS}

R. P. DILWORTH
HERBERT FEDERER
MARSHALL HALL

\author{
P. R. HALMOS \\ HEINZ HOPF \\ R. D. JAMES
}

\author{
B $\emptyset$ RGE JESSEN \\ PAUL LÉVY \\ GEORGE PÓLYA
}

\author{
J. J. STOKER \\ E. G. STRAUS \\ KÔSAKU YOSIDA
}

\section{SPONSORS}

UNIVERSITY OF BRITISH COLUMBIA

CALIFORNIA INSTITUTE OF TECHNOLOGY

UNIVERSITY OF CALIFORNIA, BERKELEY

UNIVERSITY OF CALIFORNIA, DAVIS

UNIVERSITY OF CALIFORNIA, LOS ANGELES

UNIVERSITY OF CALIFORNIA, SANTA BARBARA

OREGON STATE COLLEGE

UNIVERSITY OF OREGON

\author{
UNIVERSITY OF SOUTHERN CALIFORNIA \\ STANFORD UNIVERSITY \\ WASHINGTON STATE COLLEGE \\ UNIVERSITY OF WASHINGTON

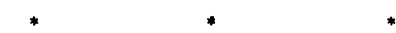 \\ AMERICAN MATHEMATICAL SOCIETY \\ NATIONAL BUREAU OF STANDARDS, \\ INSTITUTE FOR NUMERICAL ANALYSIS
}

Mathematical papers intended for publication in the Pacific Journal of Mathematics should be typewritten (double spaced), and the author should keep a complete copy. Manuscripts may be sent to any of the editors. All other communications to the editors should be addressed to the managing editor, E. F. Beckenbach, at the address given above.

Authors are entitled to receive 100 free reprints of their published papers and may obtain additional copies at cost.

The Pacific Journal of Mathematics is published quarterly, in March, June, September, and December. The price per volume (4 numbers) is $\$ 8.00$; single issues, $\$ 2.50$. Spécial price to individual faculty members of supporting institutions and to members of the American Mathematical Society: $\$ 4.00$ per volume; single issues, $\$ 1.25$.

Subscriptions, orders for back numbers, and changes of address should be sent to the publishers, University of California Press, Berkeley 4, California.

UNIVERSTTY OF CALIFORNIA PRESS • BERKELEY AND LOS ANGELES 


\section{Pacific Journal of Mathematics}

\section{Vol. 1, No. $3 \quad$ BadMonth, 1951}

R. P. Boas, Completeness of sets of translated cosines ............. 321

J. L. Brenner, Matrices of quaternions . ..................... 329

Edmond Darrell Cashwell, The asymptotic solutions of an ordinary differential equation in which the coefficient of the parameter is

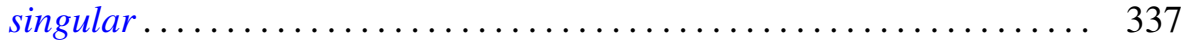

James Dugundji, An extension of Tietze's theorem ................ 353

John G. Herriot, The polarization of a lens ...................... 369

J. D. Hill, The Borel property of summability methods ............... 399

G. G. Lorentz, On the theory of spaces $\Lambda \ldots \ldots \ldots \ldots \ldots \ldots \ldots \ldots \ldots . \ldots \ldots$

J. H. Roberts and W. R. Mann, On a certain nonlinear integral equation of the Volterra type ................................. 431

W. R. Utz, A note on unrestricted regular transformations . . .......... 447

Stanley Simon Walters, Remarks on the space $H^{p} \ldots \ldots \ldots \ldots \ldots \ldots . . \ldots 55$

Hsien Chung Wang, Two theorems on metric spaces ............... 473 